\title{
Modificación química del precursor de titanio para obtener soles estables de silice - titania: Uso de acetilacetona
}

\author{
J. E. RODRÍGUEZ-PÁEZ1', A. MAFLA', G. ANDRADE¹, A.DURÁN² \\ 'Grupo CYTEMAC. Departamento de Física - FACNED, Universidad del Cauca. Popayán - Cauca/ COLOMBIA \\ ${ }^{2}$ Instituto de Cerámica y Vidrio - C.S.I.C, Cantoblanco, Madrid - España
}

\begin{abstract}
La técnica Sol-Gel se ha utilizado para sintetizar una serie de sistemas multicomponentes, entre ellos $\mathrm{SiO}_{2}-\mathrm{TiO}_{2}$. El mayor problema en la obtención de geles multicomponentes estables es la desigual velocidad de hidrólisis y condensación que presentan los alcóxidos precursores de los cationes de interés. En este trabajo se muestra cómo adicionando acetilacetona, acacH, al sistema TEOS $-\mathrm{Ti}(\mathrm{OBu})_{4}-\mathrm{H}_{2} \mathrm{O}$ se puede obtener un sol estable. Se tomaron diferentes concentraciones de los precursores de silicio y titanio y una sola concentración de acacH. Se utilizó espectroscopia infrarroja, FTIR, para identificar los grupos funcionales presentes en el sistema y además se midió regularmente la viscosidad para determinar cualitativamente el avance de la policondensación del sistema.
\end{abstract}

Palabras claves: sol-gel; silice-titania; acetilacetonato; sol estable.

Chemical modification of titanium precursor to obtain stable silica-titania sol: acetylacetone.

Sol-gel processing has become a well established technique for producing ceramic powders or glasses. This processing has been utilized to synthesize several interesting systems, e.g. the $\mathrm{SiO}_{2}-\mathrm{TiO}_{2}$ system. A major concern in the stable multicomponent geles is that the hydrolysis and condensation velocities are diferent for each precursor, TEOS and Ti $(\mathrm{OBu})_{4}$ in this work. The chemical control of these reactions is currently performed by adding complexing reagents that react with metal alkoxides at a molecular level, giving rise to new molecular precursors of different structure, reactivity and functionanality. This paper shows that stable TEOS - Ti $(\mathrm{OBu})_{4}-\mathrm{H}_{2} \mathrm{O}$ sol can be reproducibly prepared in the presence of acetylacetone. We shall then show that the acac behaves as a ligand, directly bonded to the titanium ion. Thus the formation of precipitate is avoided. Infra-red spectroscopy (FTIR) and viscosity measures were used to demostrated this behaviour of the system.

Keywords: sol-gel; silica - titania; acetylacetone; stable sol.

\section{INTRODUCCION}

El procesamiento Sol - Gel es considerado uno de los métodos más promisorios para la obtención de vidrios y cerámicos $(1,2,3)$. Utilizando este método es posible obtener cerámicos multicomponentes $(1,4)$. El sistema multicomponente estudiado en este trabajo es el de $\mathrm{SiO}_{2}-\mathrm{TiO}_{2}$ el cual presenta multiples aplicaciones $(5,6)$. Para este sistema, la obtención de un gel homogéneo multicomponente es difícil debido a que las velocidades de reacción de los precursores son diferentes (7); la velocidad de hidrólisis del $\mathrm{Ti}(\mathrm{OBu})_{4}$ es mayor que la del TEOS lo que favorece la formación de enlaces $\mathrm{Ti}-\mathrm{O}-\mathrm{Ti}$, frente a los de $\mathrm{Ti}-\mathrm{O}-\mathrm{Si}$, dando como resultado un sol micro heterogéneo (8).

El método más utilizado actualmente para favorecer la homogeneidad del sol es el de adicionar un acomplejante que reaccione con los precursores a nivel molecular para producir nuevos precursores moleculares (9). Los resultados indican que estos aditivos químicos permiten un mejor control del proceso $(10,11)$ debido a que los alcóxidos, menos electronegativos, se hidrolizan de manera preferencial mientras que los grupos acomplejantes, fuertemente ligados, son más difíciles de remover (12).

Para el sistema $\mathrm{TiO}_{2}-\mathrm{SiO}_{2}$ se han utilizado como acomplejantes ácido acetico y $\beta$ - dicetonas $(9,13)$. En el presente trabajo se utilizó la acetilacetona que presenta ligandos fuertemente quelatantes y que permitiría la formación de $\beta$-dicetonas metálicas, compuestos que contienen un grupo hidroxil que favorecería la reacción con el alcoxido de titanio $(9,13,14)$. Se comprobó cómo la presencia del acetilacetonato, acac, en el sistema silicio - Titanio permite la formación de un gel homogéneo estable durante varios días. Se utilizó espectroscopia infrarroja para caracterizar los soles y determinar la naturaleza del nuevo precursor molecular de titanio. El avance de la gelificación del sistema $\mathrm{SiO}_{2}-\mathrm{TiO}_{2}$ fue monitoreado a través de la variación de la viscosidad del sistema en función del tiempo.

\section{PROCEDIMIENTO EXPERIMENTAL.}

\subsection{Obtención de los Soles.}

Los soles se obtuvieron utilizando como precursor de silicio tetraetil-ortosilicato, TEOS -(Aldrich), y de titanio tetrabutóxido de titanio, Ti(OBu $)_{4}$ - (Aldrich). El Ti(OBu) se mezcló con etanol, la mitad del volumen que se empleo, y acetilacetona, acacH - (Aldrich), en una relación molar $\mathrm{TiO}_{2} /$ acac $=1$. Este sistema se dejó envejecer 40 minutos al término de los cuales se dio inicio paralelamente a la prehidrólisis del TEOS. Para ello se realizó la mezcla TEOS- $\mathrm{C}_{2} \mathrm{H}_{5} \mathrm{OH}-\mathrm{H}_{2} \mathrm{O}$ tomando la otra mitad del volumen de etanol y la mitad del volumen total de agua acidificada $(\mathrm{HCl} 0.1 \mathrm{~N})$ empleada. Se dejó pre-hidrolizar el TEOS durante 20 minutos, manteniendo el sistema en agitación y a continuación se combinaron las dos mezclas. Se adicionó la otra mitad de $\mathrm{H}_{2} \mathrm{O}$ y se mantuvo el sistema en agitación a 400 r.p.m. por 2 horas. Las relaciones molares utilizadas se determinaron de la expresión $\mathrm{xSi} / \mathrm{yTi}$ donde $\mathrm{x}+\mathrm{y}=1$. La tabla 1 indica las relaciones molares $\mathrm{Si} / \mathrm{Ti}$ 
estudiadas, así como el número de moles de los precursores, solventes y acomplejante utilizados. Los soles conformados se mantuvieron a temperatura ambiente, en frascos cerrados.

TABLA I. RELACIONES MOLARES [Si]/[Ti] ESTUDIADAS Y DATOS DE LOS VOLÚMENES UTILIZADOS DE ACOMPLEJANTE, PRECURSORES Y SOLVENTES PARA LA CONFORMACIÓN DE LOS SOLES.

\begin{tabular}{|c|c|c|c|c|c|}
\hline $\mathrm{Ti} / \mathrm{Si}$ & NTBT & NTEOS & NH2O / nTotal & NacacH / nTotal & NEtOH / nTotal \\
\hline $10 / 90$ & 0.0285 & 0.0032 & 1.5 & 0.9075 & 29.13 \\
\hline $20 / 80$ & 0.0260 & 0.0065 & 1.5 & 0.8070 & 27.15 \\
\hline $30 / 70$ & 0.0234 & 0.0100 & 1.5 & 0.7037 & 26.67 \\
\hline $40 / 60$ & 0.0206 & 0.0138 & 1.5 & 0.6053 & 26.56 \\
\hline $50 / 50$ & 0.0176 & 0.0177 & 1.5 & 0.5034 & 25.74 \\
\hline $60 / 40$ & 0.0145 & 0.0220 & 1.5 & 0.4000 & 25.24 \\
\hline $70 / 30$ & 0.0112 & 0.0260 & 1.5 & 0.3010 & 24.30 \\
\hline $80 / 20$ & 0.0077 & 0.0307 & 1.5 & 0.2010 & 24.59 \\
\hline $90 / 10$ & 0.0039 & 0.0362 & 1.5 & 0.1000 & 23.86 \\
\hline
\end{tabular}

nTotal: número de moles de TEOS + número de moles de TBT

N: Número de moles.

\subsection{Caracterización del Sistema.}

Se tomaron muestras de los soles sílice-titánia dos horas después de mezclados los sistemas que contenían separadamente los precursores. Estas muestras se caracterizaron utilizando espectroscopia infrarroja (espectrómetro Perkin-Elmer 580). La estabilidad del sol fue monitoreada visualmente y el avance de la gelación del sistema se monitoreo registrando los valores de viscosidad medidos a tiempos diferentes durante varias semanas, utilizando para ello un viscosímetro capilar tipo Ubbeighde calibrado bajo la norma ASTMD 495.

\section{RESULTADOS Y DISCUSIÓN.}

El sistema $\mathrm{Ti}(\mathrm{OBu})_{4}$-acac- $\mathrm{C}_{2} \mathrm{H}_{5} \mathrm{OH}$ estudiado no presentó formación de precipitado aún después de mezclar las soluciones que contenían separadamente los precursores; esta condición perduró durante el envejecimiento. Se observó cambio de coloración del sistema, dependiendo de la relación molar [Si] / [Ti], desde una suave tonalidad amarilla (baja concentración de titanio) hasta un amarillo intenso (alta concentración de titanio). Esta coloración se debe a la absorción óptica alrededor de los $330 \mathrm{~nm}$ por la transferencia de carga del ligando acac al ion Ti(IV) (7).

En la figura 1 se muestran los espectros de infrarrojo correspondientes a soles de $\mathrm{SiO}_{2}-\mathrm{TiO}_{2}$ con diferentes razones [Si]/[Ti]. Las bandas a $1465 \mathrm{~cm}^{-1}-1378 \mathrm{~cm}^{-1}$ pueden asociarse a las vibraciones de tensión de los grupos alifáticos $\mathrm{CH}_{2}$ y $\mathrm{CH}_{3}$ del precursor de titanio (15), y a otros compuestos presentes en el sistema como el etanol. El hombro a $1125 \mathrm{~cm}^{-1}$, y las bandas a $1085 \mathrm{~cm}^{-1}$ y $1049 \mathrm{~cm}^{-1}$, deben corresponder a las vibraciones Ti-O-C de los grupos butoxi ligados directamente al titanio (15). Las bandas a $2971 \mathrm{~cm}^{-1}, 2930 \mathrm{~cm}^{-1}$ y $2870 \mathrm{~cm}^{-1}$ contienen información del modo $v\left(\mathrm{CH}_{3}\right)$, simétrico y asimétrico, y del $\gamma\left(\mathrm{CH}_{2}\right)$; tanto del $\mathrm{Ti}(\mathrm{OBu})_{4}$ como del compuesto titanio-acetilacetonato que se forma en el sistema (16). El doblete de bandas a $\sim 1587 \mathrm{~cm}^{-1} \mathrm{y} \sim 1530 \mathrm{~cm}^{-1}$ corresponden a los modos vibracionales $v(\mathrm{C}=\mathrm{C})$ y $\mathrm{v}(\mathrm{C}=\mathrm{O})$. Esta división en dos bandas es una evidencia de que los ligandos acac están enlazados directamente al titanio ya que el acacH libre solo presenta una banda de absorción ancha alrededor de $1620 \mathrm{~cm}^{-1}(7,17)$. Otras bandas que se pueden asociar al complejo titanio-acac son: la de $\sim 1445 \mathrm{~cm}^{-1}$, modo vibracional combinado $\delta(\mathrm{CH})$ y $v(\mathrm{C}=\mathrm{C})$, la ubicada a $\sim 1425 \mathrm{~cm}^{-1}$, asignada al $\delta_{\mathrm{d}}\left(\mathrm{CH}_{3}\right)$, la banda intensa a $1379 \mathrm{~cm}^{-1}, \delta_{\mathrm{s}}\left(\mathrm{CH}_{3}\right), \sim 1274 \mathrm{~cm}^{-1}$, al modo combinado $v\left(\mathrm{C}-\mathrm{CH}_{3}\right)+v(\mathrm{C}=\mathrm{C}), \sim 930 \mathrm{~cm}^{-1}, v(\mathrm{C}=\mathrm{C})+v(\mathrm{C}=\mathrm{O})$ y la banda ancha centrada en $666 \mathrm{~cm}^{-1}$ que debe contener información sobre los modos $v\left(C-\mathrm{CH}_{3}\right)+$ deformación del anillo+v(Ti-O) (16). La expresión deformación del anillo hace referencia al ambiente coordinativo tipo benceno que puede presentar el compuesto titanio-acac $(16,17)$.

Al enlace Ti-O-Si se le puede asociar el pequeño hombro que aparece a $940 \mathrm{~cm}^{-1}$ (17) y la banda a $\sim 950 \mathrm{~cm}^{-1}$ al butanol generado por la hidrólisis del Ti $(\mathrm{OBu})_{4}(18)$. La banda a $880 \mathrm{~cm}^{-1}$ corresponde al etanol y la ubicada a $\sim 980 \mathrm{~cm}^{-1}$ se atribuye a la formación de especies silanol ( $\mathrm{Si}$ $\mathrm{OH})$. En el espectro [Si] / [Ti] $=50 / 50$ aparece una banda a $\sim 435 \mathrm{~cm}^{-1}$, de intensidad media, que se puede asociar a la vibración de deformación del enlace O-Si-O (19).

De acuerdo a los resultados de infrarrojo es evidente la formación en el sistema de un complejo $\mathrm{Ti}\left(\mathrm{OBu}^{\mathrm{n}}\right)_{4-\mathrm{x}}(\mathrm{acac})_{x}$ de acuerdo a la siguiente reacción:

$$
\mathrm{Ti}\left(\mathrm{OBu}^{\mathrm{n}}\right)_{4}+\mathrm{acacH} \rightarrow \mathrm{Ti}\left(\mathrm{OBu}^{\mathrm{n}}\right)_{4-\mathrm{x}}(\mathrm{acac})_{x}+\mathrm{xBuOH}
$$

Esta reacción química es coherente con la propuesta para modificaciones químicas ocasionadas por ligandos nucleofílicos hidrolizados, ácidos carboxílicos o $\beta$-dicetonas (20). Con base en lo mencionado, y considerando que los ligandos acac están directamente enlazados al titanio tal como se deriva del estudio de FTIR, se propone como posible estructura del nuevo precursor molecular de titanio la que se muestra en la figura 2.
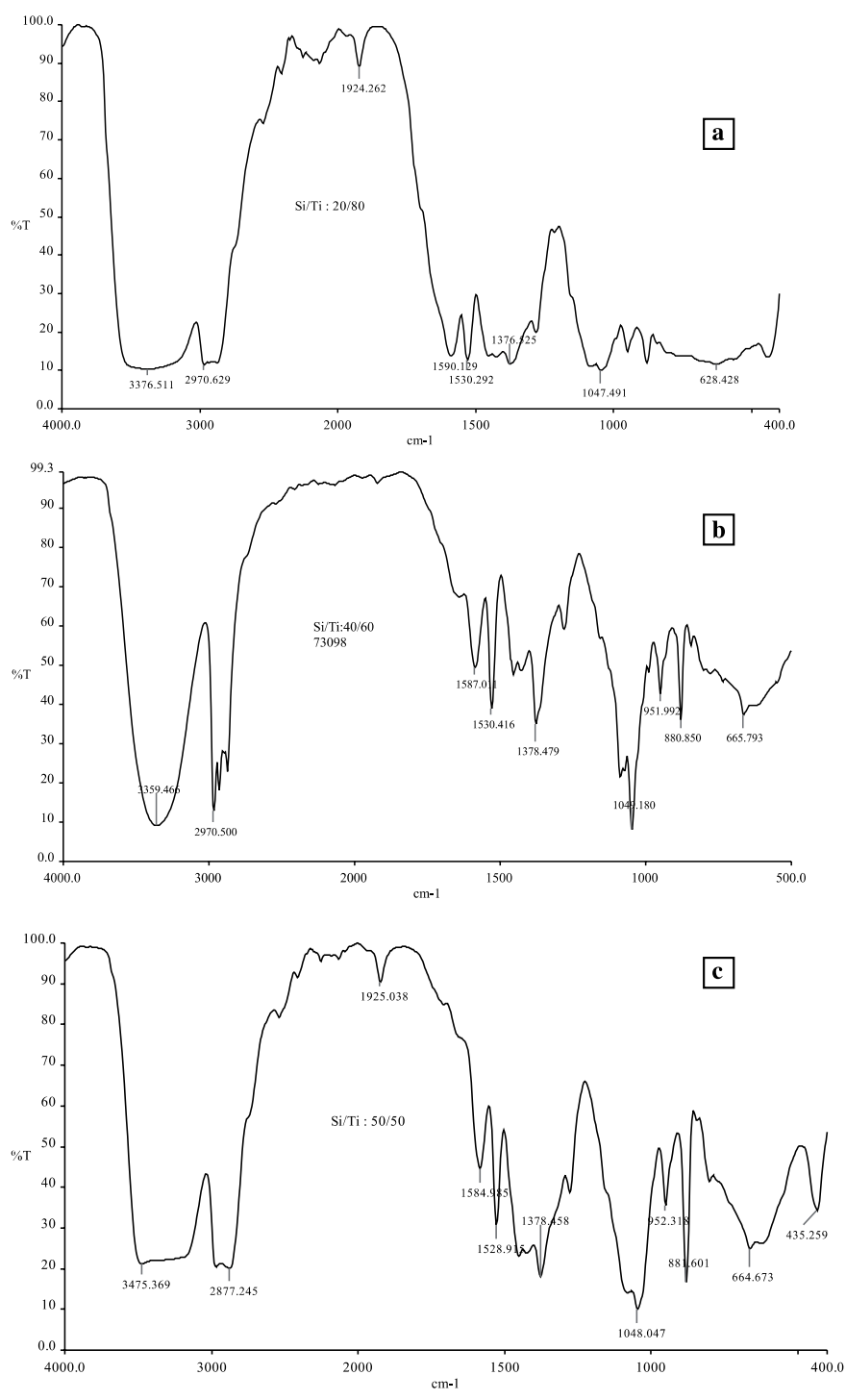

Figura 1. Espectros de infrarrojo correspondientes a concentraciones [Si]/[Ti] de 20/80 (a), 40/60 (b) y 50/50 (c). 


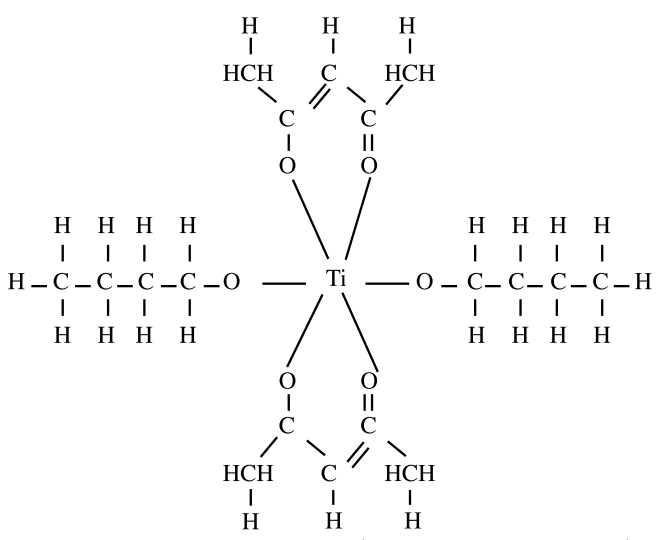

Figura 2. Estructura propuesta para el nuevo precursor molecular de titanio.

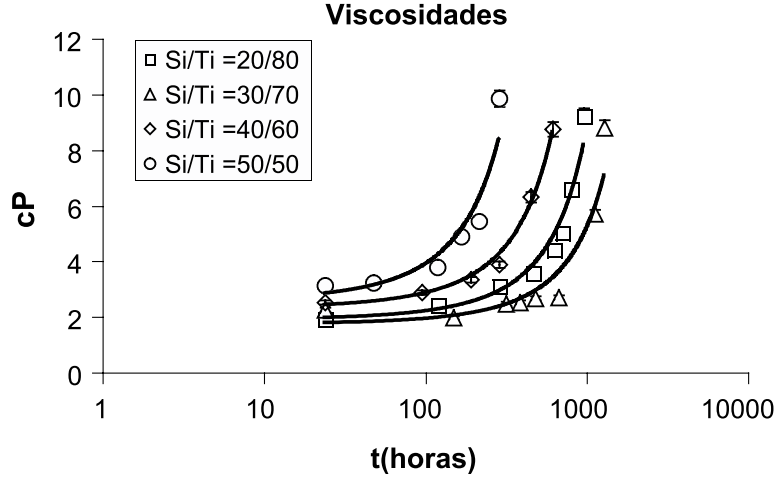

Figura 3. Variaciones de la viscosidad con el tiempo de los sistemas estudiados.

En la figura 3 se indica como varían los valores de viscosidad del sistema con el tiempo para las diferentes concentraciones [Si]/[Ti] estudiadas. En estas curvas es evidente la estabilidad de los soles. Observando cuidadosamente las curvas de viscosidad, la tendencia de desplazamiento hacia tiempos mayores del punto de gelación de los sistemas, a medida de que se disminuye la concentración de titanio, se ve alterada cuando se toman concentraciones $[\mathrm{Si}] /[\mathrm{Ti}]=40 / 60 \mathrm{y}$ 50/50 (el tiempo de gelación disminuye). Aunque la razón de acomplejamiento para todos los sistemas tiene un valor de uno, $r_{c}=1$, lo que lleva a esperar soles estables (20), parece que para relativas bajas concentraciones de $\mathrm{Ti}(\mathrm{OBu})_{4}$ se manifiesta el poder catalizador de este alcóxido sobre la condensación de los silanoles (21). Esta hipótesis se sustenta observando los espectros de FTIR (figura 1) donde para la relación $[\mathrm{Si}] /[\mathrm{Ti}]=50 / 50$ aparece la banda a $\sim 435 \mathrm{~cm}^{-1}$ asociada con la vibración de deformación del enlace O-Si-O (19).

\section{CONCLUSIONES.}

De acuerdo a los resultados obtenidos se puede concluir lo siguiente:

1.- La concentración de acetil-acetona utilizado permite obtener soles estables del sistema binario $\mathrm{SiO}_{2}-\mathrm{TiO}_{2}$. El acacH reacciona con el tetrabutóxido de titanio a nivel molecular dando origen a un nuevo precursor con una estructura diferente a la del compuesto inicial. Se propone una posible estructura para el nuevo precursor de titanio.

2. La estabilidad de los soles se extiende a varios días y se requieren varias semanas para que ellos comiencen a gelificar tal como lo indican las curvas de viscosidad del sistema. Los ligandos acac se deben comportar como reactivos de terminación ocasionando la reducción de la funcionalidad del nuevo precursor de titanio y evitando la formación de un precipitado.
3. Para concentraciones relativamente bajas de $\mathrm{Ti}\left(\mathrm{OBu}_{4}^{\mathrm{n}}\right)_{4}$ en el sistema, el alcoxido de titanio aparentemente cataliza la condensación de silanoles favoreciendo la homocondensación de especies de silicato más que la incorporación de titanio a nivel molecular. Se requiere un estudio más cuidadoso sobre la estructura del sistema $\mathrm{TiO}_{2}-\mathrm{SiO}_{2^{\prime}}$ para bajas concentraciones de titanio, con el fin de obtener más información al respecto.

\section{AGRADECIMIENTOS}

Este trabajo fue financiado a través de los proyectos COLCIENCIAS N ${ }^{\circ} 1103-05-605-93$ y VRI-Universidad del Cauca N 752 . Agradecemos a Ecopetrol-ICP la colaboración prestada con los estudios de espectroscopia infrarroja y a la red CyTED VIII.E la ayuda económica y técnica gracias a la cual A. Mafla pudo realizar su pasantía en el ICVCSIC de Madrid-España.

\section{REFERENCIAS.}

1. C. J. Brinker, G. W. Scherer, Sol-Gel Science: the physics and chemistry of sol-gel processing, Academic Press, Inc. 1990.

2. L. L. Hech, D. R. Ulrich (editores), Science of Ceramic: Chemical Processing, John Wiley \& Sons, Inc. 1986.

3. L. Klein (editora), Sol-gel technology for thin films, fibers, preforms, electronics and specialty shapes, Noyes Publications, Park Ridge, N. J., 1988.

4. H. Schimidt, "Inorganic - Organic composites by Sol-Gel techniques", J.solgel Sci. and Techn, 1, 217-231 (1994).

5. R. B. Pettit, C. S Ashley, S.T.Reed, C.J.Brinker, "Antireflective films from the sol-gel process", pp. 80-109 en sol-gel technology for thin, fibers, performs, electronics and specialty shapes" editado por L.C.Klein, Noyes publications, 1988.

6. M. Guglemi," sol gel coating on metals", J. sol-gel Sci.and Techn., 8, 443-449 (1997).

7. J. Livaje, "Synthesis, structure and applications of $\mathrm{TiO}_{2}$ gels", Mat. Res. Soc. Symp. Proc., 73, 717 - 724 (1986)

8. W.C. LaCourse, S. Kim, "Use of mixed titanium alcoxides for sol-gel processing", pp.304-310 en Science of Ceramic Chemical Processing, editors L.L. Hench y D.R. Uldrich, John Wiley \& sons, Inc ,1986.

9. C. Sanchez, J. Livaje, M. Henry, F. Babonneau,"Chemical modifications of alkoxide precursor", J. Non.- Crys.Solids, 100, 65-75 (1988).

10. A. C. Lópes, J. Gallardo, A. Durán, "Síntesis y estructura de recubrimientos híbridos de ZrO2-SiO2 obtenidos por so-gel”, Bol. Soc. Esp. Ceram.V., 40 [6] 429-434 (2001)

11. M. Aparicio, A. Durán, "Protección contra la oxidación de materiales compuestos $\mathrm{SiC}(\mathrm{C} / \mathrm{SiC})$ mediante la combinación de recubrimientos de silicatos de itrio y silice", Bol. Soc. Esp. Ceram.V., 40 [6] 441-445 (2001).

12. J.Livaje,"Molecular desing of transition metal alkoxide precursor", pp.3-32 en Chemical processing of ceramics", editado por B.I Lee, y E.J.A Poper, Marcel Dekker Inc., 1994.

13. C. Sanchez, F. Baboneeau; S. Doeuff, A. Leaustic, "Chemical Modifications of titanium alkoxide precursor", pp 77-87 en ultrastructure processing of ceramics glasses and composites (San Diego1987), 1988.

14. D.C Bradley ,R.C. Mehrotra, D.P.Gaur, Metal Alkoxide, Academic Press, New York, 1978.

15. S.Doeuff, M.Henry, C. Sanchez, J.Livaje, "Hidrolysis of titanium alkoxides: modification of the molecular precursor by acetic acid", J. Non-Cryst. Solid, 89, 206-216 (1987).

16. K.Nakamoto, Infrared and Raman Spectra of Inorganic and coordination compounds, part B, $5^{\text {th }}$ edition, John Wiley \& Sons Inc., 1997.

17. G. Socrates, Infrared Characteristic Group Frecuencies, John Wiley \& Sons, Inc., 1994.

18. M.J.Velasco, F.Rubio, J.Rubio, L.L.Oteo, "Hydrolysis of titanium tetrabutoxide study by FTIR", Spectroscopy Lett., 32 (2), 289-307 (1999).

19. M.J.Velasco, Sintesis y caracterización de ormosiles obtenidos a partir del TEOS y PDMS, Tesis doctoral Universidad autónoma de Madrid, 1999.

20. A.C.Pierre, Introduction to sol-gel processing , Kluwer Academic Publishers, 1998.

21. W.Noll, Chemie and technology der silicone, $2^{\text {nd }}$ ed. Verlag chemie, Weinheim, F. R. Germany, 1968.

Recibido: 01.02 .03

Aceptado: 30.11 .03 